\title{
Die Auswahl zukünftiger Kinder
}

\section{Tatjana Tarkian}

Online publiziert: 15 . Juni 2020

(C) Der/die Autor(en) 2020

Zusammenfassung Wie ist die Auswahl zukünftiger Kinder in moralischer Hinsicht zu beurteilen? Der Beitrag untersucht diese Frage auf der Basis eines liberalen Prinzips der reproduktiven Freiheit, welches Handlungsspielräume und Privatsphäre in Fragen der Fortpflanzung verteidigt, wenn nicht gewichtige moralische Gründe aufgezeigt werden können, welche den Handlungen der beteiligten Personen Grenzen setzen. Er beschränkt sich auf die negative Auswahl, d.h. die Auswahl von (möglichen) gesunden Kindern anstelle von zukünftigen Kindern mit Behinderung oder gesundheitlicher Beeinträchtigung. Erörtert werden selektionsbefürwortende Überlegungen, welche das Wohlergehen des zukünftigen Kindes in den Fokus rücken, und zwei grundsätzlich selektionskritische Argumente. Das erste verweist auf parentale Tugenden, welche mit der Auswahl zukünftiger Kinder unvereinbar wären. Bei dem zweiten handelt es sich um den Einwand der Diskriminierung von Menschen mit Behinderung. Das Ergebnis der Diskussion ist, dass es zukünftigen Eltern in den meisten Fällen freisteht, die Entscheidung über die Auswahl ihrer Kinder im Licht ihrer Ziele und Werte zu treffen.

Schlüsselwörter Pränataldiagnostik · Reproduktionsethik · Fortpflanzungsethik · Auswahl $\cdot$ Behinderung $\cdot$ Gesundheit $\cdot$ Eugenik

\section{Einleitung}

Mit dem Fortschritt in der Reproduktionsmedizin, Humangenetik und vorgeburtlichen Diagnostik hat sich die Geburt eines Kindes zunehmend vom zufälligen Er-

T. Tarkian $(\bowtie)$

Seminar für Philosophie, Universität Erfurt, Nordhäuser Str. 63, 99089 Erfurt, Deutschland

E-Mail: tatjana.tarkian@uni-erfurt.de 
eignis zum Ergebnis einer Kette von Entscheidungen gewandelt. ${ }^{1}$ Dieter Birnbacher spricht von der Entwicklung, ,von der Widerfahrnis zur Gestaltung, von der Kontingenz zum Arrangement, von der Heteronomie zur Autonomie“ (2017, 4); diese betrifft nicht nur Sterben und Tod, sondern gleichermaßen die Geburt. Dabei wirft die Möglichkeit der Auswahl zukünftiger Kinder zahlreiche moralische Fragen auf. Tagespolitische Bedeutung erlangten sie zuletzt mit der Markteinführung nicht-invasiver molekulargenetischer pränataler Tests (NIPT) in Deutschland, an die sich eine kontroverse öffentliche Debatte anschloss. Können Schwangere ein legitimes Interesse an der Kenntnis genetischer Merkmale des Fötus geltend machen? Auf welche Merkmale des Fötus sollten Frauen testen lassen können? Wie sind selektive Abtreibungen grundsätzlich zu beurteilen? In welchen Fällen sind sie zulässig, sind sie in manchen Fällen sogar geboten, falls sie nicht grundsätzlich in moralischer Hinsicht zu verurteilen sind?

Dieser Beitrag widmet sich der Frage, wie die Auswahl zukünftiger Kinder in moralischer Hinsicht zu beurteilen ist. Grundlage meiner Diskussion reproduktionsethischer Fragen wird ein liberales Prinzip der reproduktiven Freiheit sein, welches Handlungsspielräume und Privatsphäre in Fragen der Fortpflanzung verteidigt, wenn nicht gewichtige moralische Gründe aufgezeigt werden können, welche den Handlungen der beteiligten Personen Grenzen setzen (4). Kritiker der Praxis der Pränataldiagnostik (PND) und des selektiven Schwangerschaftsabbruchs haben wiederholt verschiedene Einwände geltend gemacht. Betrachten werde ich hier den Hinweis auf parentale Tugenden und ein moralisches Ideal der Familie, welches eine Zurückweisung von zukünftigen Kindern mit bestimmten Merkmalen verbiete (5) und den Einwand der Diskriminierung von Menschen mit Behinderung (6). Die Frage wird sein, ob diese Einwände Aspekte erfassen, denen das moralische Gewicht zukommt, das nötig wäre, um den Gehalt der reproduktiven Freiheit in entsprechender Weise zu beschränken. Dabei geht es mir nicht um die Frage nach rechtlichen Beschränkungen der reproduktiven Freiheit, sondern um die Konturen der moralischen Handlungsspielräume mit Blick auf die Familienplanung. Zuletzt wende ich mich Überlegungen zu, die auf das Wohlergehen des zukünftigen Kindes abheben und dieses der elterlichen Verantwortung als Maßstab vorgeben (7). Konsequentialisten haben mit Blick auf die Auswahl zukünftiger Kinder zahlreiche wohlfahrtsorientierte Überlegungen vorgestellt, deren Würdigung die Möglichkeiten dieses Diskussionsbeitrags übersteigt. Ich werde mich auf die Frage konzentrieren, ob es einen moralischen Grund für die Auswahl eines gesunden zukünftigen Kindes anstelle eines zukünftigen Kindes mit Beeinträchtigung gibt. Zunächst soll ein sehr kurzer Blick auf die aktuellen Möglichkeiten der nicht-invasiven genetischen Pränataldiagnostik die praktische Dimension des Themas illustrieren (2). Anschließend wird geklärt, was mit der Auswahl zukünftiger Kinder gemeint ist (3).

\footnotetext{
1 Titelgebend war diese Entwicklung für das bekannte Werk From Chance to Choice (Buchanan et al. 2000).
}

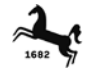




\section{Nicht-invasive genetische Pränataldiagnostik (NIPD)}

Das Spektrum vorgeburtlicher Untersuchungen hat sich in den letzten Jahren erweitert. Als neues Instrument der genetischen Frühdiagnostik ist die nicht-invasive pränatale Diagnostik (NIPD) an die Seite invasiver Verfahren getreten. Das Blut der Schwangeren enthält schon im ersten Schwangerschaftstrimester Bruchstücke zellfreier fetaler DNA plazentaren Ursprungs. Als dies in den neunziger Jahren erkannt wurde, galt die Entwicklung von Tests auf genetische Eigenschaften des Fötus durch die Untersuchung des mütterlichen Bluts nur noch als eine Frage der Zeit. ${ }^{2}$ Nicht-invasive pränatale Tests (NIPT) werden seit 2011 in den USA und seit 2012 in Deutschland von verschiedenen Anbietern vertrieben. Die erste Generation der Tests richtete sich auf die Früherkennung von Aneuploidien, und dabei zunächst auf die Identifikation der drei häufigsten Trisomien: Trisomie 21 (Down-Syndrom), Trisomie 18 (Edwards-Syndrom) und Trisomie 13 (Pätau-Syndrom). Mittlerweile wird auch die Suche nach geschlechtschromosomalen Mono- und Trisomien angeboten. Das diagnostische Potenzial der NIPT ist aber nicht auf den Nachweis chromosomaler Fehlverteilungen beschränkt. Über eine Probe des Bluts der Schwangeren ist ab der zehnten Schwangerschaftswoche der Zugriff auf die vollständigen genetischen Daten des Fötus technisch möglich geworden. ${ }^{3}$ Es liegen Veröffentlichungen über geglückte Gesamtgenomanalysen des Ungeborenen vermittels des mütterlichen Bluts vor (vgl. Lo et al. 2010; Kitzman et al. 2012). Die zweite Generation der NIPT kann auch Mikrodeletionen identifizieren. Dazu zählen das 5p-Deletionssyndrom, das Angelman- und das Prader-Willi-Syndrom. Betroffene zeigen neben Entwicklungsverzögerungen und organischen Fehlfunktionen geistige Behinderungen unterschiedlichen Grades. Es gibt weitere Mikrodeletionssyndrome, und für einzelne ist ebenfalls ein Test verfügbar (so für das 22q11.2-Mikrodeletions- oder DiGeorgeSyndrom). Die jüngste Testgeneration, in Deutschland seit 2019 angeboten, richtet sich nun auch auf den Nachweis monogener Erkrankungen: die zystische Fibrose, spinale Muskelatrophie, Sichelzellanämie, die Thalassämien. Es gibt mehrere Tausend monogener Erkrankungen, und für viele sind die genetischen Ursachen aufgeklärt. Man darf daher annehmen, dass weitere Tests entwickelt werden.

Die Ergebnissicherheit vorgeburtlicher Bluttests ist hoch, ihre Kosten sind bereits deutlich gefallen, und mit ihnen wird der Einsatz invasiver Methoden rückläufig, die später in der Schwangerschaft erfolgen, belastend und mit einem geringen Fehlgeburtsrisiko behaftet sind. Entsprechend hat der Gemeinsame Bundesausschuss, wie von vielen erwartet, im September 2019 beschlossen, nicht-invasive Tests auf autosomale Trisomien ,für die Anwendung bei Schwangerschaften mit besonderen Risiken“ in den Leistungskatalog der gesetzlichen Krankenversicherung aufzunehmen. Im Fokus der öffentlichen Debatte in Deutschland stand der Test auf fetale Trisomie 21 und dessen Finanzierung auf der Grundlage des Solidarprinzips.

\footnotetext{
2 Vgl. Lo et al. 1997. Die nicht-invasive Pränataldiagnostik an fetalen Zellen aus mütterlichem Blut (und nicht an zellfreier fetaler DNA) war bereits zuvor Gegenstand einer kurzen Verlautbarung der Deutschen Gesellschaft für Humangenetik (vgl. Kommission für Öffentlichkeitsarbeit und ethische Fragen der Gesellschaft für Humangenetik 1993).

3 In Deutschland ist eine Gesamtgenomanalyse des Fötus durch $§ 15$ GenDG untersagt.
} 
Ganz neu sind die Fragen nicht, vor die uns der Fortschritt in der medizinischen Genetik stellt. Die PND hat seit gut einer Generation ihren festen Platz in der Schwangerschaftsbegleitung. Mit immer besserer Ultraschalltechnik können Auffälligkeiten in Wachstum und Entwicklung sowie organische Fehlbildungen frühzeitig erkannt werden, oft zum Wohl des Ungeborenen. Invasive Methoden der genetischen PND, erstmals 1966 angewandt, werden seit den achtziger Jahren vermehrt genutzt - und zwar zuletzt in recht hohem Ausmaß: In den Jahren vor der Einführung der NIPD wurde in Deutschland in fast jeder zehnten Schwangerschaft, die zu einer Geburt führte, eine invasive Diagnostik durchgeführt. Dem wachsenden Wissen um genetische Ursachen von Erkrankungen und Behinderungen stehen allerdings noch immer die fehlenden Möglichkeiten an ursächlicher Therapie gegenüber. Im Fall eines positiven Befunds bleibt daher nur die Entscheidung zwischen einem selektiven Schwangerschaftsabbruch und der Fortsetzung der Schwangerschaft mit der Aussicht auf die spätere Nutzung der verfügbaren Therapien und Fördermaßnahmen. Genaue Prognosen über die Entwicklungsmöglichkeiten oder den Krankheitsverlauf des zukünftigen Kindes sind dabei oft nicht möglich. Schwangere müssen daher unter Ungewissheit entscheiden.

Die Praxis der PND wird seit langem von einem kritischen Echo begleitet. Neuartige Fragen wirft die Methode der NIPT nicht auf. Aber ihr nicht-invasiver Charakter könnte die Routinisierung der PND befördern, welche Kritiker aus den Reihen der Behindertenbewegung und Autoren aus den Disability Studies häufig in den Fokus ihrer Kritik stellen. Die bereits zahlreichen Testziele und das Potenzial der Methode könnten die Auswahl zukünftiger Kinder noch erweitern, und daher lohnt es sich, die Frage ihrer moralischen Bewertung erneut zu stellen.

\section{Was ist mit der Auswahl zukünftiger Kinder gemeint?}

Mit der Auswahl zukünftiger Kinder ist die Entscheidung für ein (mögliches) zukünftiges Kind zuungunsten eines anderen (möglichen) zukünftigen Kindes gemeint - also der Versuch, ein (mögliches) zukünftiges Kind durch ein anderes (mögliches) zukünftiges Kind zu ,ersetzen“. ${ }^{4}$ Geschehen könnte dies entweder durch seine erst in der Zukunft liegende Zeugung oder durch die Entscheidung, eine Schwangerschaft mit einer bestimmten bereits befruchteten Eizelle zu erzeugen und nicht mit einer anderen. Bei der Präimplantationsdiagnostik wird die Auswahl zeitgleich vorgenommen: Es liegen mehrere befruchtete Eizellen vor, und es wird entschieden, mit einem bestimmten Embryo (oder mit mehreren Embryos) eine Schwangerschaft zu erzeugen und Embryos mit bestimmten genetischen Merkmalen nicht in den Uterus einer Frau zu transferieren. Auch im Rahmen gewöhnlicher Kinderwunschbehandlungen wird gewöhnlich eine Auswahl unter den befruchteten Eizellen getroffen, wenn auch nach äußerlichen Kriterien: Für den Transfer in den Uterus werden wohlgeformte

\footnotetext{
4 Vgl. ähnlich Wilkinson 2012, 2. Unter einem möglichen Kind ist eines zu verstehen, das in der Zukunft geboren werden könnte und noch nicht gezeugt ist. Mit einem zukünftigen Kind ist bereits gezeugtes gemeint, das geboren werden könnte. Ich blende hier die Frage aus, zu welchem Zeitpunkt das zukünftige Kind individuiert ist.
} 
Zellen ausgewählt und asymmetrische zurückgewiesen. Meist wird die selektive Fortpflanzung aber zeitversetzt verfolgt. Paare können sich nach einem pränataldiagnostischen Befund entscheiden, eine Schwangerschaft zu beenden, und später versuchen, erneut ein Kind zu zeugen. Oder eine Frau kann entscheiden, nicht zum jetzigen Zeitpunkt schwanger zu werden, sondern erst später, etwa nach Abschluss ihrer Berufsausbildung oder mit einem anderen Partner. Die Beispiele der aufgeschobenen Familiengründung und der unabgeschlossenen Partnersuche zeigen, dass die Auswahl zukünftiger Kinder auch außerhalb eines reproduktionsmedizinischen Kontexts erfolgen kann. Sie ist hier in moralischer Hinsicht unproblematisch. Es entspricht jedenfalls dem vorherrschenden Verständnis der reproduktiven Freiheit, dass die Partnerwahl, der Kinderwunsch, der Zeitpunkt der Familiengründung und die Kinderzahl im Normalfall in den persönlichen Entscheidungsspielraum fallen.

Es gibt also verschiedene Fälle selektiver Fortpflanzung, die Motive dürften dabei unterschiedlich gelagert sein, und einige Fälle sind offensichtlich in keiner Weise zu beanstanden. Es soll im Folgenden nur um die Auswahl von zukünftigen Kindern gehen, die nicht von einem bestimmten genetischen Merkmal mit Krankheitswert oder einer durch PND diagnostizierten Entwicklungsstörung betroffen sind: die Auswahl von gesunden Kindern anstelle von Kindern mit gesundheitlichen Beeinträchtigungen oder Normabweichungen, wie sie eine geistige Behinderung darstellt. Einer solchen „,negativen Auswahl“ steht die Auswahl von Kindern gegenüber, die in ihren körperlichen oder geistigen Eigenschaften mehr als durchschnittlich sind, anstelle von gewöhnlichen gesunden Kindern. Transhumanisten befürworten eine solche ,positive Auswahl“, wenn sie auf freiwilliger Basis und in individueller, d.h. nicht staatlich gesteuerter Form geschieht; sie könnte aus ihrer Sicht durch Enhancement ergänzt werden, also durch die Verbesserung der genetischen Ausstattung eines Individuums durch gezielte Eingriffe, vielleicht mittels der Genchirurgie. Eine klare Abgrenzung zwischen negativer Auswahl - mit dem Ziel der Vermeidung von Gesundheitsstörungen oder Behinderungen - und positiver Auswahl - mit dem Ziel der Optimierung oder Perfektionierung - ist schwer zu ziehen. Darauf haben schon viele hingewiesen. Ein Abgrenzungsversuch würde eine Erörterung der Begriffe der Gesundheit, Krankheit und Behinderung erfordern, die hier nicht versucht werden soll. Es muss daher genügen zu sagen, dass es hier nur um die negative Auswahl gehen soll, also um Entscheidungen gegen zukünftige Kinder mit Merkmalen, die als diagnostische Ziele der PND oder NIPD genannt wurden: Trisomien, durch Mikrodeletionen verursachte geistige Behinderungen, monogene Erkrankungen oder Entwicklungsstörungen.

Selektive Fortpflanzungsentscheidungen haben die Existenz zukünftiger Personen zur Folge und wirken sich damit auf ihre Identität aus (sie sind ,identity-affecting“"). Zeugt jemand ein Kind mit Partner A und nicht mit B, oder zeugt er es mit Partner A hier und heute und nicht in drei Monaten - von seiner Entscheidung hängt ab, welches mögliche Kind in der Zukunft existieren wird. Solche Entscheidungen nennt 
Derek Parfit in seinem klassischen Beitrag zum Thema „different people choices“.5 Sie können das moralische Urteilen auf eine harte Probe stellen. Davon wird später noch zu sprechen sein (vgl. Abschn. 7).

\section{Das liberale Prinzip der reproduktiven Freiheit}

Ein zentrales Ideal freiheitlicher Gesellschaften ist das der individuellen Freiheit. ${ }^{6}$ Es entwirft die Vision von Menschen, die über ihr Leben so weit wie möglich selbst bestimmen und es im Licht ihrer Vorstellungen des Guten gestalten. Der Begriff der reproduktiven Freiheit bezeichnet Handlungsspielräume einer Person, die ihre auf die Fortpflanzung bezogenen Entscheidungen betreffen. ${ }^{7}$ Der Wert der reproduktiven Freiheit lässt sich auf der Grundlage verschiedener ethischer Konzeptionen unterschiedlich begründen. Da ihre Begründung hier nicht im Vordergrund der Betrachtung steht, mag es genügen zu sagen, dass es gut für den Menschen ist, Lebenspläne zu entwerfen, selbstbestimmt Entscheidungen zu treffen und sein eigenes Leben gestalten oder wenigstens auf seinen Verlauf Einfluss nehmen zu können. Mit der Partnerwahl, der Gründung einer Familie, aber auch mit der Entscheidung gegen eigene Kinder kann ein Leben maßgeblich geprägt werden. Die Entscheidung für Kinder begründet im Normalfall eine lang andauernde Fürsorgebeziehung. Es ist gut, Handlungsspielräume in Fragen zu haben, welche die Fortpflanzung betreffen. Das ist auch unstrittig. Strittig ist nur die Frage nach der Reichweite der reproduktiven Freiheit.

Ich favorisiere ein liberales Prinzip der reproduktiven Freiheit als Grundlage der Diskussion reproduktionsethischer Fragen. Dieses Prinzip verteidigt Freiheitsspielräume und Privatsphäre in Fragen der Fortpflanzung, wenn nicht gewichtige moralische Gründe aufgezeigt werden können, welche den Handlungen von Personen Grenzen setzen. ${ }^{8}$ Dem Prinzip wohnt die Annahme inne, dass fortpflanzungsbezogene Handlungen moralisch zulässig - also weder falsch noch geboten oder supererogatorisch - sind, solange nicht moralische Gründe vorliegen, welche gegen ihre Zulässigkeit sprechen. Stephen Wilkinson hat dies die Präsumption der Zulässigkeit genannt. ${ }^{9}$ Die eigentliche Begründungsaufgabe liegt darin, den substanziellen Gehalt der reproduktiven Freiheit angesichts all der neuen technischen, medizinischen und sozialen Möglichkeiten genau zu bestimmen. Die Präsumption der Zulässigkeit führt mithin zu einer negativen Methode: Reproduktive Optionen sind im Licht der

\footnotetext{
5 Vgl. Parfit 1984, 356. Parfit unterscheidet weiter zwischen Entscheidungen, deren Ergebnis die Zahl der existierenden Personen nicht verändert (,,same number choices“) und solchen, deren Folgen sowohl die Identität als auch die Zahl der existierenden Personen beeinflussen (,different number choices“). Entscheidungen der letzten Art sind Gegenstand der Populationsethik.

6 Ich greife in diesem Abschnitt auf einen früheren Beitrag zurück, vgl. Tarkian 2018.

7 Ronald Dworkin hat ausführlich für das Recht auf reproduktive Autonomie argumentiert, dies aber im rechtsethischen Kontext, der hier ausgeblendet bleibt. Vgl. Dworkin 1993, Kapitel 6.

8 Vgl. ähnlich Bennett und Harris 2007, 202, und in ähnlicher Absicht, wenn auch nicht gleichlautend Robertson 1996, 16.

9 Vgl. Wilkinson 2012, 13. In der Formulierung der Annahme weiche ich von Wilkinson ab.
} 
Frage zu betrachten, ob moralische Gründe gegen sie sprechen. Lassen sich keine solchen Gründe erkennen, dürfen sie als zulässig gelten.

Das liberale Prinzip wurde vereinzelt als allzu permissiv oder undifferenziert zurückgewiesen. Diese Kritik kann auf einem Missverständnis beruhen. Welche Reichweite die reproduktive Freiheit hat, ist zunächst eine offene Frage. Das Prinzip ist mindestens so sehr methodischer Art, wie es substanzieller Art ist. Seine Anhänger können sehr permissive, sie können aber auch restriktivere Konzeptionen dieser Freiheit verteidigen. Der liberale Charakter des Prinzips zeigt sich sicherlich darin, dass die Begründungshürden für die Zurückweisung der Zulässigkeit individueller Fortpflanzungsentscheidungen hoch sind. Aus liberaler Sicht gebietet es der Respekt vor der Autonomie von Personen, ihre Entscheidungen auch dann zu tolerieren, wenn diese in den Augen mancher nicht unbedingt begrüßenswert oder ratsam erscheinen. Dies gilt insbesondere eingedenk der modernen Pluralität von Wertorientierungen und unterschiedlichen Vorstellungen hinsichtlich der Familie. Die Zeugung eines Kindes in einer lesbischen Paarbeziehung oder der Vertrag mit einer „Tragemutter“ stellen aus der Perspektive mancher Wertorientierungen ein Ärgernis dar. Das allein reicht aus liberaler Sicht nicht, um diese Praktiken moralisch zu verurteilen. Dafür müssten stärkere Gründe geltend gemacht werden können.

Charakteristisch für eine liberale Position im Anschluss an Mill ist die Auffassung, dass die Freiheit dort ihre Grenzen findet, wo Dritten Schaden zugefügt wird oder Schaden absehbar ist. ${ }^{10}$ Ich möchte die moralischen Gründe, welche die Zulässigkeit einer Fortpflanzungsentscheidung in Frage stellen, allerdings nicht vorab auf solche eingrenzen, welche am besten unter der Kategorie des Schadens zu fassen sind. Die besondere Dimension reproduktiver Entscheidungen ergibt sich daraus, dass sie eine besondere Klasse von Betroffenen haben - nämlich zukünftige Kinder. Die Folgen elterlicher Entscheidungen für ihre Kinder sind in individualethischer Hinsicht sicherlich von zentraler Bedeutung für die Beurteilung der Konturen elterlicher Freiheitsspielräume. Daneben sind es aber auch Gerechtigkeitserwägungen, welche zur Einschränkung der reproduktiven Freiheit geltend gemacht werden können. Würden Menschen mit angeborener Behinderung durch pränatale Gentests und Abtreibungsentscheidungen diskriminiert, wäre das ein starker Grund zur Beschränkung der negativen Auswahl. Daher verdient der Einwand der Diskriminierung beachtet zu werden (vgl. Abschnitt 6).

\section{Parentale Tugenden und das moralische Ideal der Familie}

Autorinnen und Autoren aus den Disability Studies beurteilen genetische Pränataldiagnostik und die Praxis des selektiven Schwangerschaftsabbruchs mehrheitlich sehr kritisch. Der sozialwissenschaftlichen Orientierung der Forschungsrichtung entsprechend ist es Sozialkritik, die dabei im Mittelpunkt steht. Mithin rücken nicht individuelle Fallkonstellationen und Entscheidungen von Schwangeren in den Fokus. Stattdessen werden Ungleichheit, Machtstrukturen und Machtgefälle, Diskrimi-

10 Vgl. Mill 1859. Diese Sicht kommt auch in Robertsons Position zum Ausdruck (vgl. 1996, 16 sowie 40f.). 
nierung, Vorurteile und deren unterstellte Einbettung in eine medizinische Routine thematisiert, der sich zu entziehen aus Sicht der Kritiker für Schwangere oder Paare mit Kinderwunsch äußerst schwierig, ja fast kaum möglich ist. Damit schreibt der Diskurs der Disability Studies in seiner Stoßrichtung die Kritik fort, die seit den achtziger Jahren von Aktivisten aus der Behindertenbewegung gegen die Praxis der vorgeburtlichen Diagnostik vorgebracht wurde. Diese bezog sich bekanntlich und letztlich auch folgerichtig ebenfalls auf konsequentialistische Beiträge in der angewandten Ethik, in denen, zu Unrecht oder nicht, diskriminierende Urteile über das Leben von Menschen mit Behinderung ausgemacht wurden (vgl. dazu Abschnitt 7).

Es ist nicht möglich, dieser Kritik hier umfassend Rechnung zu tragen. Ausgeblendet bleiben insbesondere viele sozialkritische Aspekte - nicht, weil ich sie für irrelevant halte, sondern weil sie sich schlecht im Rahmen eines Beitrags erörtern lassen, der nach den moralischen Konturen der reproduktiven Freiheit fragt. Ich will dagegen zwei Einwände herausgreifen, welche wiederholt gegen die Auswahl zukünftiger Kinder vorgetragen wurden, unter anderem auch von Stimmen aus den Disability Studies. Es handelt sich dabei um spezifische Einwände gegen die selektive Fortpflanzung, deren Anhänger das Recht von Frauen, sich zu einem konkreten biographischen Zeitpunkt gegen eine Schwangerschaft zu entscheiden, als Bestandteil ihrer Fortpflanzungsfreiheit betonen. Die Einwände richten sich also nicht gegen den Schwangerschaftsabbruch als Mittel der selektiven Fortpflanzung.

Einer dieser Einwände ist tugendethischer Art. So heißt es, die Annahme eines Kindes und die elterliche Liebe seien nicht an Bedingungen zu knüpfen. Es sei eine parentale Tugend, alle Kinder gleichermaßen willkommen zu heißen und zu lieben. Hier wird wahlweise vom Ideal der bedingungslosen Liebe, der unbedingten Hingabe (,,ideal of unconditional devotion“, Asch und Wasserman 2005, 202) oder Hinwendung (,,commitment“) gesprochen. Adrienne Asch hat diese Überlegung schon vor Längerem vorgebracht (vgl. 2000). Gemeinsam mit David Wasserman schreibt sie:

[T] he opposition to parental selectivity is fundamentally nonconsequentialist.

What is wrong about such selectivity is not, or not principally, that it may have harmful consequences, but that it reflects, and expresses, an impoverished conception of parenthood and families (Asch und Wasserman 2005, 202).

David Wasserman betrachtet das Ideal der bedingungslosen Hinwendung (,,ideal of unconditional welcome“) als Grundlage „eines der stärksten Einwände“ (2009, 324) gegen die vorgeburtliche Auswahl zukünftiger Kinder:

$[\mathrm{P}]$ reconception and prenatal selection are morally problematic because they are incompatible with the ideal of unconditional welcome to which prospective parents should aspire. This ideal extends the unqualified commitment that parents try to maintain towards their existing children to the onset of the parental project; the point at which a couple or individual decides to bring a child into the family. Under this ideal, any eligibility requirement for family membership, beyond the prospect of a life worth living and the capacity to form intimate relationships, is not compatible with the moral ideal of the family. [...] The (quasi-) random process of fertilization deserves deference not because it is natural, but because it precludes a selectivity that prospective parents ideally should not exercise (Wasserman 2009, 324). 
Zuneigung und Liebe haben ihren Platz in einem Ideal der Familie. Bedingungslose Liebe ist freilich ein hohes Ideal, von dem vielleicht auch nicht klar ist, ob es tatsächlich unter allen Umständen anzustreben ist. Das selektionskritische Argument erscheint daher, so denke ich, noch am aussichtsreichsten, wenn es auf einem Ideal der unbedingten Hinwendung und nicht auf einem der unbedingten Liebe basiert. Es gehört zu den elterlichen Tugenden, seine Hinwendung zum Kind nicht an Bedingungen zu knüpfen und den Platz des Kindes in der Familie nicht von seinen spezifischen Merkmalen oder seiner Erfüllung elterlicher Erwartungen abhängig zu machen. Gute Eltern wenden sich ihren Kindern zu, ohne Bedingungen zu stellen. Die entscheidende Frage ist aber, warum dieses Elternideal hin zum Beginn des elterlichen Projekts ausgedehnt werden sollte, wie Asch und Wasserman es ausdrücken - zur Entscheidung, eine Familie zu gründen (vgl. 2005, 203). Warum sollte unbedingte Hinwendung nicht nur eine Tugend aktualer, sondern auch eine Tugend potenzieller Eltern zukünftiger Kinder sein? ${ }^{11}$

Um das Ideal der unbedingten Hinwendung in ein günstiges Licht zu stellen, lenken seine Fürsprecherinnen und Fürsprecher die Aufmerksamkeit auf den besonderen Charakter der Familie. ${ }^{12}$ Diese unterscheidet sich als sozialer Verbund von einem Verein, der auf freiwilliger Assoziation beruht, und einem Club, der die Zugehörigkeit durch die Auswahl neuer Mitglieder durch die Clubmitglieder regelt. Paarbeziehungen werden durch Zuneigung und beiderseitige Zustimmung gestiftet, und eine Selektivität ist hier angemessen. Die Beziehung, die Eltern durch die Zeugung und mit der Geburt eines Kindes stiften, ist in dieser Hinsicht einzigartig. Anders als Paarbeziehungen, die auch von einem Ideal des Wohlwollens und der Liebe geprägt sind, sind biologische Familienrelationen nicht auflösbar. Eltern haben Verpflichtungen gegenüber ihren Kindern, die aus ihrer Fürsorgeverantwortung resultieren. ${ }^{13}$ Übernehmen sie diese Verantwortung, so sind sie nur dann gute Eltern, wenn sie ihre Zuwendung und Hingabe nicht von spezifischen Merkmalen ihrer Kinder abhängig machen.

Damit ist die Frage aber nicht beantwortet. Einem geborenen Kind aufgrund seiner Merkmale die Hinwendung zu versagen ist etwas anderes, als ein zukünftiges Kind ,zurückzuweisen“. ${ }^{14}$ Hinwendung und Liebe erfordern ein Gegenüber. Mögliche Kinder sind kein Gegenüber. ${ }^{15}$ Auch zukünftige Kinder, die sich in einem frühen Entwicklungsstadium befinden, können keine Zurückweisung erfahren. Allerdings verfehlen diese Hinweise den Kern des tugendethischen Arguments. Dieses zielt darauf, dass ein selektiver Blick auf (mögliche) zukünftige Kinder mit dem morali-

\footnotetext{
11 Diese kritische Frage haben andere ebenfalls gestellt. Vgl. Shakespeare 2014, 126 und Wilkinson 2012, Kapitel 2.

12 Vgl. Asch und Wasserman 2005, 200 ff.; Wasserman 2009, 326. Vgl. auch Kittay 2000.

13 Können sie diese Verantwortung nicht schultern, ist es geboten, dass ein anderer in die Fürsorgebeziehung zum Kind eintritt.

14 Mehrfach erlangten Fälle kommerzieller Tragemutterschaft Publizität, bei denen das auftraggebende Paar die Annahme des Kindes nach seiner Geburt wegen einer Behinderung oder genetischen Erkrankung verweigert hat. Diese Fälle erregten zu Recht Missbilligung und werfen auch ein ungünstiges Licht auf das Arrangement der Tragemutterschaft.

15 Dies sei erwähnt, da Wasserman in der zuletzt zitierten Passage die präkonzeptionelle Auswahl in seine Kritik einschließt.
} 
schen Ideal der Familie unverträglich ist. Auswahl möge für Paarbeziehungen und Clubs angemessen sein, aber nicht für die Familie: ${ }^{16}$

We contend that impairments, and other characteristics viewed as "burdensome and irksome," will rarely defeat reasonable hopes and dreams for rearing a child or preclude rewarding relationships with the child. And we then suggest that abandoning a parental project in the face of such characteristics, or making that project contingent on the absence of such characteristics, is inconsistent with the commitment to welcome and nurture any child the parents have, a commitment we regard as the moral foundation of the family (Asch und Wasserman 2005, 203).

Handelt es sich beim Ideal der unbedingten Hinwendung um die moralische Grundlage der Familie? Aus meiner Sicht ist das moralische Fundament der Familie durch selektive Fortpflanzungsentscheidungen jedenfalls nicht grundsätzlich gefährdet. Das tugendethische Argument basiert auf einer besonderen Konzeption parentaler Tugenden, die zu würdigen, deren Verbindlichkeit für alle aber fraglich ist. In der Familie werden individuelle Vorstellungen der persönlichen Lebensgestaltung entwickelt, erprobt und, wenn alles gut geht, realisiert. Mill sprach im Zusammenhang mit der Erprobung von Vorstellungen des Guten von Lebensformexperimenten $(1859,261)$. Gerade die Familiengründung ist ein Projekt, das als solch ein Experiment verstanden werden muss. Das Ideal der bedingungslosen Hinwendung gehört zu Vorstellungen des Guten, die man schätzen und anderen vorleben, die zu teilen man aber aus liberaler Sicht von anderen nicht einfordern kann. Es gibt, mit anderen Worten, Raum für vernünftigen Pluralismus hinsichtlich moralischer Ideale der Familie. Daher liefert das tugendethische Argument keinen Grund, die negative Auswahl grundsätzlich zu missbilligen. Doch es ist als grundsätzlicher Einwand gedacht - mit einer Einschränkung: Der selektive Blick wird (jedenfalls von Wasserman) nur dann kritisiert, wenn er beim zukünftigen Kind nach mehr sucht als nach der Aussicht auf ein ,lebenswertes Leben mit der Fähigkeit, vertraute Beziehungen zu anderen aufzunehmen“" (2009, 324).

Der Hinweis auf elterliche Tugenden fehlt auch in der öffentlichen Diskussion nicht. Hier verbirgt er sich manchmal hinter dem irreführenden Slogan, es gebe kein Recht auf ein gesundes Kind. Ein solches Recht kann es gewiss nicht geben. Die eigentliche Frage ist aber, ob Schwangere verpflichtet sind, eine Auswahl gesunder zukünftiger Kinder zu unterlassen, oder ob Paare schlechte zukünftige Eltern wären, wenn sie sich diagnostischer Methoden bedienen möchten. Beides kann mit dem Ideal der bedingungslosen Hinwendung nicht gezeigt werden.

\section{Der Einwand der Diskriminierung}

Ein Einwand begleitet die moderne Schwangerschaftsbegleitung schon seit Jahrzehnten: Die Praxis der Pränataldiagnostik und der selektiven Abtreibung sei Ausdruck

16 Wasserman bezieht seine Kritik folgerichtig auch auf die Auswahl (möglicher) zukünftiger Kinder mit Merkmalen wie Gehörlosigkeit. 
einer diskriminierenden Haltung gegenüber Menschen mit Behinderung. Auch als „expressivistischer Einwand“ (expressivist objection) bezeichnet, stellt er ein Kernelement der Kritik aus den Disability Studies dar (vgl. Parens und Asch 2000). Er wird auf unterschiedliche Weise vorgebracht und oft nicht präzisiert. Marsha Saxton formuliert ihn wie folgt:

The message at the heart of widespread selective abortion on the basis of prenatal diagnosis is the greatest insult: some of us are "too flawed" in our very DNA to exist; we are unworthy of being born. This message is painful to confront (Saxton 2017, 85).

Diese Fassung spiegelt die persönliche Betroffenheit Saxtons deutlich wider. Sie wirft aber auch die Fragen auf, was es denn genau heißt, dass eine Praxis eine Botschaft aussende, und ob man wirklich dieses generell abwertende Urteil über Menschen mit Behinderung hinter individuellen Entscheidungen gegen die Fortsetzung einer Schwangerschaft vermuten müsste (vgl. Tarkian 2018, $169 \mathrm{ff}$.). Immerhin dürften die Motive für einen Schwangerschaftsabbruch (egal ob selektiver Art oder nicht) vielgestaltig und auf die jeweils persönliche Lebenssituation bezogen sein. Diese Fragen haben ihre Berechtigung. Doch es darf nicht übersehen werden, dass der Einwand der Diskriminierung in erster Linie in sozialkritischer Absicht vorgebracht wird. Er richtet sich vorrangig gegen den Einsatz der PND als Routineverfahren, gegen ihre breite Akzeptanz, mithin auch gegen kassenfinanzierte nicht-invasive Tests auf fetale Trisomien und gegen die „Normalisierung“ selektiver Abbrüche. All dies reflektiere verbreitete ablehnende Haltungen oder das aus Sicht der Kritikerinnen diskriminierende Urteil, dass das Leben von Menschen mit Behinderung schlechter sei als ein Leben ohne Beeinträchtigungen. In Adrienne Aschs Worten lautet der Einwand der Diskriminierung, dass ,Pränataldiagnostik die Überzeugung widerspiegelt oder einschließt, dass Kinder mit Beeinträchtigungen sich selbst, ihren Eltern und der Gesellschaft eine Last sind" (Asch und Wasserman 2005, 172). Dieses diskriminierende Urteil werde durch medizinische und soziale Praktiken zum Ausdruck gebracht und gleichzeitig fortgeschrieben.

Der Einwand der Diskriminierung differenziert nicht entlang von Merkmalen oder Beeinträchtigungen, die durch PND identifiziert werden könnten. In seiner pauschalen Form ist seine Plausibilität schwer zu beurteilen. Eine Antwort liegt aber auf der Hand. Liberale werden das Angebot der PND nur dann als Erweiterung der reproduktiven Freiheit verstehen, wenn die individuelle Entscheidung zur Diagnostik selbstbestimmt und nicht unter dem Druck von verbreiteten diskriminierenden Urteilen über das Leben von Menschen mit Behinderung getroffen wird. Entscheidungen können nur dann autonom sein, wenn sie nicht durch äußere Einflüsse bestimmt werden und die inneren Bedingungen der Handlungsfähigkeit erfüllt sind. Die Befürwortung des Angebots der Pränataldiagnostik ist daher daran gebunden, dass die Entscheidung zur Diagnostik wohlerwogen und wohlinformiert erfolgt. Um Vorurteilen oder Fehleinschätzungen über den Lebensalltag von Menschen mit Behinderung entgegenzuwirken, kann etwa in der Beratungspraxis Kontakt zu Betroffenen angeboten oder hergestellt werden. Insgesamt liegt die überzeugendste Antwort auf die dem Einwand innewohnende Sozialkritik in der Überwindung der sozialen Diskriminierung von Menschen mit Behinderung. Ein wichtiger Schritt wäre hier ein 
deutlicher politischer Wille zur Durchsetzung der im Übereinkommen über die Rechte von Menschen mit Behinderungen spezifizierten Rechte. Können zukünftige Eltern zuversichtlich sein, dass ihre Kinder in einer Gesellschaft ihren Weg finden, die das Ziel der Inklusion engagiert verfolgt, dann sind die äußeren Zwänge beseitigt, die vielleicht die Entscheidung für ein Kind mit Behinderung erschweren.

Die fehlende Differenzierung ist aus meiner Sicht eine deutliche Schwäche des Einwands (vgl. Tarkian 2018). Er erklärt die mit einem Schwangerschaftsabbruch verfolgte negative Auswahl pauschal zum Ausdruck diskriminierender Haltungen. Seine Anfangsplausibilität variiert aber mit möglichen Merkmalen zukünftiger Kinder, gegen die selegiert werden könnte. Es gibt einige schwere erbliche Stoffwechselerkrankungen, die bislang nicht ursächlich therapierbar sind und zu einer deutlich reduzierten Lebenserwartung der Betroffenen führen. Die Sicht, dass das Angebot pränataler Gentests auf entsprechende Merkmale Ausdruck diskriminierender Haltungen ist, scheint ebenso wenig begründet wie eine grundsätzliche Ablehnung der PND.

Es ist das Verdienst der Behindertenbewegung und der Disability Studies, wirksam für die Rechte von Menschen mit Behinderung gekämpft und die Geschichte ihrer Diskriminierung nachgezeichnet zu haben. Erfahrungen der Ausgrenzung sind für viele leider immer noch alläglich und die Defizite mit Blick auf das Ziel der Inklusion offenkundig. Und so ist auch die Haltung der Wachsamkeit verständlich, mit der Fortschritte in der Humangenetik und vorgeburtlichen Diagnostik verfolgt werden. Allerdings besteht aus meiner Sicht grundsätzlich kein Widerspruch zwischen der Gleichberechtigung von Menschen mit Behinderung sowie einer Kultur der Anerkennung und dem Angebot der PND. Es ist offen, warum die negative Auswahl zukünftiger Kinder ganz grundsätzlich Ausdruck der Diskriminierung von Menschen mit Merkmalen sein sollte, gegen die pränatal selegiert wird. Damit wird nicht bestritten, dass vorgeburtliche Auswahl in bestimmten sozialen Kontexten Ausdruck von Diskriminierung sein kann. Was geschlechtsspezifische Auswahl in patriarchal geprägten Kulturen betrifft, ist das sicherlich der Fall. Daher kann ein Plädoyer für die Freiheit, selektive Fortpflanzungsentscheidungen zu treffen, nur überzeugend sein, wenn es mit der Kritik sozialer Benachteiligung von Menschen mit Behinderung einhergeht.

\section{Das Wohlergehen des zukünftigen Kindes}

Sind Handlungen zu beurteilen, welche die Existenz von Personen zur Folge haben, liegt es nahe, Überlegungen anzustellen, die auf ihr Wohlergehen oder ihre Lebensqualität bezogen sind. Ist die Auswahl möglicher Kinder eine Option, stellt sich nicht nur die Frage der Bewertung, sondern auch die nach interpersonalen Vergleichen von Wohlergehen. Hier handelt es sich um ein schwieriges Terrain. Es schließt sich die Frage an, welches handlungsleitende Prinzip angesichts der vergleichenden Bewertung der Folgen von Handlungen zu verteidigen ist, welche zur Existenz unterschiedlicher Personen führen. Weder der Vielzahl der entsprechenden moraltheoretischen Fragen noch der Komplexität der bisherigen Debatte kann hier Rechnung getragen werden. Ich möchte mich auf zwei Fragen beschränken. Erstens: 
Kann es moralische Gründe geben, die dafür sprechen, die Existenz einer zukünftigen Person zu verhindern? Zweitens: Spricht etwas für negative Auswahl? Genauer: Gibt es moralische Gründe, mögliche gesunde Kinder zukünftigen Kindern mit Beeinträchtigung vorzuziehen? Oder ist die Entscheidung zwischen einem zukünftigen Kind mit und einem ohne Beeinträchtigung eine moralisch neutrale Wahl?

Laura Purdy hat argumentiert, wir hätten die Pflicht zu versuchen, unseren Kindern ein in einem minimalen Sinne befriedigendes Leben (,,a minimally satisfying life“, 1996, 45) zu ermöglichen. Paare mit Kinderwunsch hätten mithin die Verantwortung, es ihren Kindern zu ersparen, mit einer sehr gravierenden gesundheitlichen Einschränkung ihren Start ins Leben zu nehmen. Wissen sie um ihr hohes Risiko, ein genetisches Merkmal mit Krankheitswert weiterzugeben, hätten sie die moralische Pflicht, sich testen zu lassen. Bei fehlenden Aussichten des zukünftigen Kindes auf eine hinreichende subjektive Lebensqualität sehen viele die Entscheidung gegen das Leben als begründet an. ${ }^{17}$ In manchen Fällen sei eine solche Entscheidung nicht nur zulässig, sondern lobenswert. ${ }^{18}$ Deutlicher fällt das Urteil von Rebecca Bennett und John Harris aus:

Where it is rational to judge that an individual would not only not have a worthwhile life, but would have a life so bad that it would be a cruelty rather than a kindness to bring it into existence, then we have not only powerful reasons not to make such choices ourselves, but also powerful moral reasons to object to those choices when made by others (Bennett und Harris 2002, 323).

Urteile darüber, welche Bedingungen für eine subjektive Lebensqualität jenseits der angenommenen Schwelle erfüllt sein müssten, dürften auseinandergehen. ${ }^{19}$ Doch dies steht der Sicht nicht im Wege, dass in gravierenden Fällen moralische Gründe für eine Entscheidung gegen das Leben sprechen. In diesen seltenen Fällen geht es darum, ein schweres Übel von jemandem abzuwenden, welches auf keine andere Weise abgewendet werden kann als dadurch, dass seine Existenz verhindert wird. Auch Nonkonsequentialisten halten hier eine Entscheidung gegen das Leben zumindest für zulässig.

Schwieriger ist die moralische Bewertung der negativen Auswahl. Es soll hier um Fälle gehen, bei denen sich die Frage der Auswahl zwischen einem möglichen gesunden Kind und einem zukünftigen Kind mit einer Erkrankung oder Behinderung stellt, die einem lebenswerten Leben nicht im Wege steht, aber mit Beeinträchtigungen verbunden ist. ${ }^{20}$ Manche Konsequentialisten meinen, dass es moralisch kritisierbar ist, wissentlich ein später krankes oder behindertes Kind auszutragen, wenn die Möglichkeit besteht, stattdessen später ein anderes, gesundes Kind zur Welt zu bringen. Einige meinen, es sei moralisch falsch, ein zukünftiges Kind mit Behinderung einem möglichen gesunden Kind vorzuziehen. John Harris hat die-

\footnotetext{
17 Vgl. stellvertretend für viele Buchanan et al. 2000, Kapitel 6, 232 ff.; McMahan 2005, 142f. und Glover 2006.

18 Vgl. Kitcher 1997, 237 (in der deutschen Übersetzung: 263).

19 Vgl. in diesem Sinne auch Glover 2001, 433.

20 Es geht also um ,same number choices“, vgl. Parfit 1984, 356.
} 
se Sicht wiederholt verteidigt. Er illustriert sie mit dem Beispiel der angeborenen Gehörlosigkeit:

[T] o choose to have a deaf child, for instance, is analogous to not curing curable deafness in a child. Just as the deaf child denied the cure is harmed by this decision, so the child with incurable deafness is harmed by the choice to bring him to birth (Bennett und Harris 2007, 206). ${ }^{21}$

Sein Urteil bezieht sich auch auf angeborene Blindheit, körperliche Beeinträchtigungen oder von Mikrodeletionen herrührende geistige Behinderungen. Mit diesen Merkmalen geboren zu werden stellt, so Harris, eine Schädigung der Betroffenen dar. Um dies zu untermauern, verteidigt Harris eine Konzeption nicht personenbezogenen Schadens (impersonal harm, 1998, $109 \mathrm{ff}$.). Gehörlos, blind oder in anderer Weise behindert zu sein, heiße, sich in einer ,harmed condition“ zu befinden. Da die Entscheidung für ein gehörloses, blindes oder behindertes Kind einen solchen Schaden erzeuge, der vermeidbar ist, sei sie moralisch falsch.

Jonathan Glover möchte Harris nicht in dem Punkt folgen, dass die Entscheidung für ein gehörloses Kind dieses Kind schädige. ${ }^{22}$ Doch er stimmt mit ihm darin überein, dass die Wahl zwischen einem Kind mit und einem ohne Behinderung keine moralisch neutrale Entscheidung sei (vgl. 2006, 26). Dies erklärt sich auf dem Hintergrund seiner Definition von Behinderung. Behinderung umfasse, so Glover, „eine Funktionseinschränkung, welche (entweder aus sich heraus oder, was häufiger der Fall ist, in Verbindung mit sozialer Benachteiligung) die Fähigkeit zum menschlichen Gedeihen beeinträchtigt" (2006, 9). Die Entscheidung für ein Kind, dessen Fähigkeit zum Gedeihen beeinträchtigt ist, ist aus seiner Sicht kritikwürdig.

Auch Peter Singer legt seiner Position die Annahme zugrunde, „,dass ein Leben ohne Behinderung besser ist als ein Leben mit Behinderung“ (Singer 2013, 96, vgl. auch 297). Diese Bewertung ist so weitverbreitet, dass man hier von der ,Standardauffassung“" sprechen kann. Das Handlungsprinzip des Utilitarismus führt in Verbindung mit ihr zum Urteil, dass die Entscheidung gegen ein zukünftiges Kind mit Behinderung richtig ist, wenn dieses durch ein gesundes Kind ,ersetzt“ werden könnte. ${ }^{23}$ Autorinnen und Autoren aus den Disability Studies haben die Standardauffassung wiederholt als vorurteilsbehaftet, diskriminierend oder kränkend kritisiert. Fürsprecher der Standardauffassung beeilen sich $\mathrm{zu}$ betonen, und dies sicher $\mathrm{zu}$ Recht, dass Urteile über die Lebensqualität von Personen keine Urteile über ihren moralischen Wert implizieren und daher nicht diskriminierend sind (vgl. McMahan 2005, 148; Singer 2013, 297). Die Standardauffassung wird dabei oft mit dem Hinweis verteidigt, dass die Suche nach therapeutischen Ansätzen oder verbesserten

\footnotetext{
21 Vgl. auch Bennett und Harris 2002, 324f. Rebecca Bennett teilt diese Sicht nicht. Ihre abweichende Position ist in beiden Beiträgen dargelegt.

22 Vgl. Glover 2006, 25. In einem früheren Beitrag hat er die Konzeption nicht personenbezogenen Schadens aber wohlwollend beurteilt, vgl. Glover 2001, $443 \mathrm{f}$.

23 Um Missverständnisse auszuschließen, betonen Konsequentialisten oft, dass ihr Urteil in ,different number choices“ anders ausfällt. Geht es um die definitiv letzte Schwangerschaft in der Familienplanung, so spräche im Fall einer moderaten Behinderung des zukünftigen Wunschkindes kein moralischer Grund für den Abbruch. Denn mit der Entscheidung für das Kind wird ein lebenswertes Leben erzeugt (vgl. z. B. Bennett und Harris 2002, 332).
} 
Therapien die beste praktische Demonstration ihrer Richtigkeit wäre. Wäre ein Leben mit Behinderung nicht schlechter als eines ohne Beeinträchtigung, so heißt es, dann gäbe es ja keinen Grund, seinen Zustand mit medizinischen Mitteln verändern $\mathrm{zu}$ wollen.

Sicherlich zielt die Suche nach Therapien darauf, die Lebensqualität von Menschen mit Behinderung zu erhöhen. Das Ziel wird oft auch erreicht. Dennoch lassen es zwei Überlegungen fraglich erscheinen, ob die Standardauffassung zur Grundlage einer moralischen Kritik der Auswahl von zukünftigen Kindern mit Behinderung gemacht werden sollte. Erstens fehlt es ihr an der erforderlichen Belastbarkeit. Zum einen handelt es sich bei der Sicht, dass ein Leben ohne Behinderung für den Betroffenen besser ist als ein Leben mit Behinderung, in doppelter Hinsicht um eine Verallgemeinerung. Unter dem Begriff der Behinderung versammeln sich recht unterschiedliche Merkmale: Sinnesbeeinträchtigungen, körperliche und geistige Behinderung und sozial-emotionale Entwicklungsstörungen. Die generalisierende Rede von „Behinderung“ oder „Beeinträchtigung“ findet sich in vielen Beiträgen. Oft lässt sie sich auch kaum vermeiden. Geht es um interpersonale Vergleiche von Lebensqualität, sind Verallgemeinerungen aber kritisch zu sehen. Weiter wäre die Standardauffassung nicht plausibel, wenn man sie als die Behauptung verstehen wollte, dass die Lebensqualität einer jeden möglichen Person ohne Behinderung D besser ist als die Lebensqualität einer (beliebigen) zukünftigen Person mit D. Eher ist sie so zu verstehen, dass die Behinderung D die Lebensqualität des Betroffenen mit einiger Wahrscheinlichkeit negativ beeinflusst (vgl. McMahan 2005, 143 f.). Das ist mit Blick auf manche Behinderungen (oder Erkrankungen, etwa die zuvor genannten monogenen Erkrankungen) wohl plausibel. Jedoch lässt sich Lebensqualität meist positiv beeinflussen. Zum anderen zeugen viele Aussagen von Menschen mit angeborenen Beeinträchtigungen von einer hohen Lebenszufriedenheit, und wenn Defizite benannt werden, dann oft in anderen Bereichen, als Menschen ohne Behinderung sie vermuten würden. Der unfall- oder krankheitsbedingte Verlust einer Funktion wird von Betroffenen meist zunächst als großes Übel beklagt. Hingegen beurteilen manche Menschen mit angeborenen Sinnesbeeinträchtigungen ihre Funktionseinschränkung nicht als Defizit - anders, als Harris annimmt. Insgesamt erscheint die Standardauffassung somit über Gebühr vereinfacht. Differenzierungen sind nötig, und es gibt offenkundig viele Fälle, in denen sich keine zuverlässige komparative Bewertung des Wohlergehens zukünftiger Personen mit und ohne Behinderung machen lässt.

Zweitens ist grundsätzlich offen, ob Handlungen im Feld der Reproduktion unter einem moralischen Imperativ der Steigerung oder gar Maximierung des Wohlergehens zukünftiger Personen stehen. Das utilitaristische Handlungsprinzip zu kritisieren ist hier nicht der Platz. Wer es auf fortpflanzungsbezogene Entscheidungen beziehen will, wird nicht nur die negative Auswahl befürworten. Er muss sich auch für eine fortpflanzungsperfektionistische Position starkmachen, wie sie Julian Savulescu verteidigt. Gemäß dem von ihm vorgeschlagenen ,Prinzip der wohltätigen Fortpflanzung“ sollten Paare oder einzelne Fortpflanzungswillige von ihren möglichen Kindern das auswählen, von dem nach dem verfügbaren Informationsstand anzunehmen ist, dass es das bestmögliche Wohlergehen haben wird (2001, 415). Geboten sei daher die Auswahl von möglichen Kindern mit nützlichen generellen 
Eigenschaften, wozu Savulescu hohe Intelligenz zählt. Mir scheint, es handelt sich hier nicht um eine moralische Verpflichtung.

\section{Schluss}

Soweit ich sehe, lassen Überlegungen, die das Wohlergehen zukünftiger Personen in den Blick nehmen, in den meisten Fällen keine gewichtigen moralischen Gründe erkennen, welche die negative Auswahl gebieten würden. In diesen Fällen sind sowohl die Entscheidung für als auch gegen ein zukünftiges Kind mit Behinderung zulässig. Somit steht es den zukünftigen Eltern frei, diese Entscheidung im Licht ihrer Ziele und Werte zu treffen.

Weder der Hinweis auf eine Diskriminierung von Menschen mit Behinderung noch das Ideal der bedingungslosen Hinwendung sind geeignet, grundsätzliche moralische Einwände gegen die Nutzung der Pränataldiagnostik und die selektive Fortpflanzung zu begründen. Der Schwangerschaftsabbruch als Mittel der selektiven Fortpflanzung konnte hier nicht gesondert bewertet werden. Im Mittelpunkt der Betrachtung stand die negative Auswahl. Dabei wurde die schwierige Frage ausgeblendet, ob dem Interesse zukünftiger Eltern an der Kenntnis der genetischen Ausstattung des zukünftigen Kindes Grenzen gesetzt sind - und wenn ja, welche und wie sie sich begründen lassen. Ebenfalls offen bleiben musste die forschungsethische Frage, wie die Entwicklung weiterer pränataler Tests zu beurteilen ist.

Funding Open Access funding provided by Projekt DEAL.

Open Access Dieser Artikel wird unter der Creative Commons Namensnennung 4.0 International Lizenz veröffentlicht, welche die Nutzung, Vervielfältigung, Bearbeitung, Verbreitung und Wiedergabe in jeglichem Medium und Format erlaubt, sofern Sie den/die ursprünglichen Autor(en) und die Quelle ordnungsgemäß nennen, einen Link zur Creative Commons Lizenz beifügen und angeben, ob Änderungen vorgenommen wurden.

Die in diesem Artikel enthaltenen Bilder und sonstiges Drittmaterial unterliegen ebenfalls der genannten Creative Commons Lizenz, sofern sich aus der Abbildungslegende nichts anderes ergibt. Sofern das betreffende Material nicht unter der genannten Creative Commons Lizenz steht und die betreffende Handlung nicht nach gesetzlichen Vorschriften erlaubt ist, ist für die oben aufgeführten Weiterverwendungen des Materials die Einwilligung des jeweiligen Rechteinhabers einzuholen.

Weitere Details zur Lizenz entnehmen Sie bitte der Lizenzinformation auf http://creativecommons.org/ licenses/by/4.0/deed.de.

\section{Literatur}

Asch, Adrienne. 2000. Why I Haven't Changed My Mind about Prenatal Diagnosis: Reflections and Refinements. In Prenatal Testing and Disability Rights. Hrsg. Erik Parens und Adrienne Asch, 234-258. Washington, D.C.: Georgetown University Press.

Asch, Adrienne, David Wasserman. 2005. Where is the Sin in Synecdoche? Prenatal Testing and the Parent-Child Relationship. In Quality of Life and Human Difference: Genetic Testing, Health Care, and Disability. Hrsg. David Wasserman, Jerome Bickenbach, Robert Wachbroit, 172-216. Cambridge: Cambridge University Press. 
Bennett, Rebecca, John Harris. 2002. Are There Lives Not Worth Living? When is It Morally Wrong to Reproduce? In Ethical Issues in Maternal-Fetal Medicine. Hrsg. Donna L. Dickenson, 321-334. Cambridge: Cambridge University Press.

Bennett, Rebecca, John Harris. 2007. Reproductive Choice. In The Blackwell Guide to Medical Ethics. Hrsg. Rosamond Rhodes, Leslie P. Francis, Anita Silvers, 201-219. Oxford, Malden: Blackwell.

Birnbacher, Dieter. 2017. Tod. Berlin: de Gruyter.

Buchanan, Allen, Dan W. Brock, Norman Daniels, Daniel Wikler. 2000. From Chance to Choice: Genetics and Justice. Cambridge: Cambridge University Press.

Dworkin, Ronald. 1993. Life's Dominion: An Argument about Abortion, Euthanasia, and Individual Freedom. New York: Knopf. Deutsch: Die Grenzen des Lebens: Abtreibung, Euthanasie und persönliche Freiheit. Reinbek: Rowohlt, 1994.

Glover, Jonathan. 2001. Future People, Disability, and Screening. In Bioethics. Hrsg. John Harris, 429444. Oxford: Oxford University Press.

Glover, Jonathan. 2006. Choosing Children: Genes, Disability, and Design. Oxford: Clarendon.

Harris, John. 1998. Clones, Genes, and Immortality: Ethics and the Genetic Revolution. Oxford, New York: Oxford University Press.

Kitcher, Philip. 1997. The Lives to Come: The Genetic Revolution and Human Possibilities. New York: Simon \& Schuster. Deutsch: Genetik und Ethik: Die Revolution der Humangenetik und ihre Folgen. Übers. Thorsten Schmidt, Barbara Schaden. München: Luchterhand, 1998.

Kittay, Eva Feder, Leo Kittay. 2000. On the Expressivity and Ethics of Selective Abortion for Disability: Conversations with My Son. In Prenatal Testing and Disability Rights. Hrsg. Erik Parens, Adrienne Asch, 165-195. Washington, D.C.: Georgetown University Press.

Kitzman, Jacob O. et al. 2012. Noninvasive Whole-Genome Sequencing of a Human Fetus. Science Translat. Medicine 4 (137): 137ra76.

Kommission für Öffentlichkeitsarbeit und ethische Fragen der Gesellschaft für Humangenetik e. V. 1993. Gegenwärtiger Stand der Diskussion zur nicht-invasiven Pränataldiagnostik von Chromosomenstörungen an fetalen Zellen aus mütterlichem Blut. Medizinische Genetik 5: 347-348.

Lo, Y.M. Dennis et al. 1997. Presence of Fetal DNA in Maternal Plasma and Serum. Lancet 350 (9076): 485-487.

Lo, Y.M. Dennis et al. 2010. Maternal Plasma DNA Sequencing Reveals the Genome-Wide Genetic and Mutational Profile of the Fetus. Science Translat. Medicine 2 (61): 61ra91.

McMahan, Jeff. 2005. Preventing the Existence of People with Disabilities. In Quality of Life and Human Difference: Genetic Testing, Health Care, and Disability. Hrsg. David Wasserman, Jerome Bickenbach, Robert Wachbroit, 142-171. Cambridge: Cambridge University Press.

Mill, John Stuart. 1859. On Liberty. In: The Collected Works of John Stuart Mill, Vol. XVIII. Essays on Politics and Society Part I. Hg. John M. Robson, 212-310. Toronto, London: University of Toronto Press, Routledge and Kegan Paul, 1977.

Parens, Erik und Adrienne Asch (Hrsg.). 2000. Prenatal Testing and Disability Rights. Hastings Center Studies in Ethics. Washington, D.C.: Georgetown University Press.

Parfit, Derek. 1984. Reasons and Persons. Oxford: Clarendon Press.

Purdy, Laura M. 1996. Genetics and Reproductive Risk: Can Having Children Be Immoral? In: Laura M. Purdy. Reproducing Persons: Issues in Feminist Bioethics, 39-49. Ithaca, London: Cornell University Press.

Robertson, John. 1996. Children of Choice: Freedom and the New Reproductive Technologies. Princeton, N.J.: Princeton University Press.

Savulescu, Julian. 2001. Procreative Beneficence: Why We Should Select the Best Children. Bioethics 15 (5-6): 413-426.

Saxton, Marsha. 2017. Disability Rights and Selective Abortion. In The Disability Studies Reader. 5. Aufl. Hrsg. Lennard J. Davis, 73-86. New York, London: Routledge.

Shakespeare, Tom. 2014. Disability Rights and Wrongs Revisited. 2. Aufl. London, New York: Routledge.

Singer, Peter. 2013. Praktische Ethik. 3., rev. und erw. Auflage. Stuttgart: Reclam.

Tarkian, Tatjana. 2018. Pränatale Diagnostik und der Einwand der Diskriminierung. In Die Perfektionierung des Menschen? Hrsg. Katharina Waldner und Thomas Bahne, 161-177. Münster: Aschendorff.

Wasserman, David. 2009. Ethical Constraints on Allowing or Causing the Existence of People with Disabilities. In Disability and Disadvantage. Hrsg. Kimberley Brownlee und Adam Cureton, 319351. Oxford: Oxford University Press.

Wilkinson, Stephen. 2012. Choosing Tomorrow's Children: The Ethics of Selective Reproduction. Oxford: Clarendon Press. 